\title{
Aneurisma coronario, hallazgo en el síndrome coronario agudo con supradesnivel del segmento ST
}

\author{
Coronary aneurysm, finding in STEMI
}

Esteban Martín Quarchioni' ${ }^{1}$ María Cecilia Licheri' ${ }^{1}$ Luis Gerardo', Alberto Licheri²

\section{RESUMEN}

Los aneurismas de las arterias coronarias fueron descritos por primera vez por Morgagni en 1761 en un enfermo de sífilis. Se trata de una patología infrecuente, con una incidencia aproximada de entre el 1 y $2 \%$ en nuestro medio. Su principal importancia, desde el punto de vista clínico, es la posibilidad de producir infartos por trombosis o espasmo y ruptura. Se conoce muy poco sobre su evolución, pronóstico y tratamiento 1

Se comunica el caso de un paciente que presenta síndrome coronario agudo con elevación del segmento ST (SCACEST) y al revascularizar se observa la presencia de aneurisma gigante en la arteria descendente anterior. Se revisa la literatura con el objetivo de actualizar la información al respecto.

Palabras clave: aneurisma, SCACEST, infarto, ectasia.

\section{ABSTRACT}

The aneurysms of the coronary arteries were first described by Morgagni in 1761 in a patient with syphilis. It is an infrequent pathology, with an approximate incidence of between 1 and $2 \%$ in our environment, its main importance, from the clinical point of view, is the possibility of producing infarcts due to thrombosis or spasm and rupture. Very little is known about its evolution, prognosis and treatment. 1

We report the case of a patient with acute coronary syndrome with st segment elevation (STEMI) and revascularization, the presence of giant aneurysm in the anterior descending artery is observed, the literature is reviewed in order to update the information in this regard

Keywords: aneurysm, STEMI, infarction, ectasia

Revista Argentina de Cardioangiología Intervencionista 2020;11(1):32-34. https://doi.org/10.30567/RACI/202001/0032-0034

\section{CASO CLÍNICO}

Paciente masculino de 67 años, hipertenso, diabético tipo II, dislipémico, medicado, no controlado (vidagliptina, metformina, atorvastatina, valsartán) acude a la guardia de nuestro nosocomio refiriendo dolor precordial de tipo opresivo, de reposo, intensidad 8/10, de 4 hs de evolución, irradiado a epigastrio y miembros superiores, acompañado de estado nauseoso. El examen clínico cardiovascular no mostró signos de anormalidad. En electrocardiograma (ECG) evidenció: ritmo sinusal, frecuencia cardiaca de $100 \mathrm{lat} / \mathrm{min}$, onda q patológica de v1 a v4, supradesnivel del ST de v1 a v5. Niveles iniciales de CPK MB fueron elevados. Se medicó en ese momento al paciente con: nitroglicerina $(10 \mathrm{~g} / \mathrm{min} \mathrm{ev})$, heparina ( $5000 \mathrm{U} \mathrm{ev})$, ácido salicílico ( $250 \mathrm{mg} / \mathrm{masticado})$, clopidogrel $(600 \mathrm{mg} / \mathrm{vo})$ se llamó de urgencia al Servicio de Hemodinamia. Se realizó cinecoronariografía, que informó: tronco de la coronaria izquierda de mediano calibre, permeable, sin lesiones; arteria descendente anterior (ADA) ocluida en su tercio proximal; arteria circunfleja $(\mathrm{ACx})$ de gran calibre, tipo ectásica, dominante, permeable, sin lesiones; arteria coronaria derecha (ACD) hipoplásica, permeable, sin lesiones (Figura 1). Se decidió realizar angioplastia de urgencia, se utilizó catéter guía $3.5\left(\mathrm{BX}^{\circ}\right)$, se cateterizó el ostium de la coronaria izquierda, se atravesó una guía floppy 0.014 (Choice) po-

1. Médico Staff del Servicio de Hemodinamia y Cirugía Cardiovascular

2. Jefe del Servicio de Hemodinamia y Cirugía Cardiovascular. Servicio de Cardiología Sanatorio Diagnóstico. Santa Fe. Argentina.

$\triangle$ Correspondencia: Esteban Quarchioni. Tel: 0342 154494055.e_quarchioni@ yahoo.com.ar

Los autores no declaran conflictos de intereses

Recibido: 30/11/2019| Aceptado: 31/01/2020 sicionándola distalmente en la ADA, se predilató con balón $2,5 \times 20 \mathrm{~mm}\left(\right.$ Maverick $\left.^{\circ}\right)$, consiguiendo la apertura del vaso. Se observó aneurisma gigante en la unión del tercio proximal con el tercio medio (Figura 2) y, de acuerdo con la disponibilidad de materiales del Servicio de Urgencia en ese momento, se optó por la utilización de un stent $3.5 \times 38$ (Waltz ${ }^{\circ}$ (Figura 3), impactándolo a $20 \mathrm{~atm}$. Con la angiografía control se observó resolución de la lesión, con revascularización del vaso tratado, sin signos de complicación y flujo final TIMI III (Figura 4).

Al reingreso a Unidad Coronaria se observó onda q de vl a v4, desaparición del supradesnivel del ST, onda t- de vl a v5, al examen físico sin signos de falla de bomba, y clínicamente desapareció el dolor precordial. Se medicó con valsartán $80 \mathrm{mg} / d i ́ a$, bisoprolol $10 \mathrm{mg} / d i ́ a$, atorvastatina $40 \mathrm{mg} /$ día, aspirina $100 \mathrm{mg} /$ día, clopidogrel $75 \mathrm{mg} /$ día, se decidió continuar con heparina ev 18.000 u/kg/día, insulina según glucemia.

El ecocardio-Doppler a las 48 hs informó: VI dimensiones normales, AI $43 \mathrm{~mm}$, RAo $36 \mathrm{~mm}$, FEVI conservada, hipoquinesia de segmentos apicales con adelgazamiento de los mismos, compatible con aneurisma de la punta, disfunción diastólica grado I, imagen hipoecoica de $17 \times 17 \mathrm{~mm}$, fija, compatible con trombo apical.

Evolución clínica favorable, se decidió el alta sanatorial al cuarto día de internación con el tratamiento antes nombrado, continuó con anticoagulación pero de manera oral con rivaroxabán $20 \mathrm{mg} / \mathrm{día}$.

En los controles clínicos seriados a los 3, 6 y 9 meses posterior al alta, el paciente refirió encontrarse estable, sin haber presentado síntomas relacionados con su enfermedad coronaria (niega angina, palpitaciones, disnea, síncopes), no ha requerido internaciones ni concurrido a guardias por síntomas relacionados y realiza actividad física aeróbica diaria, sin limitaciones. 


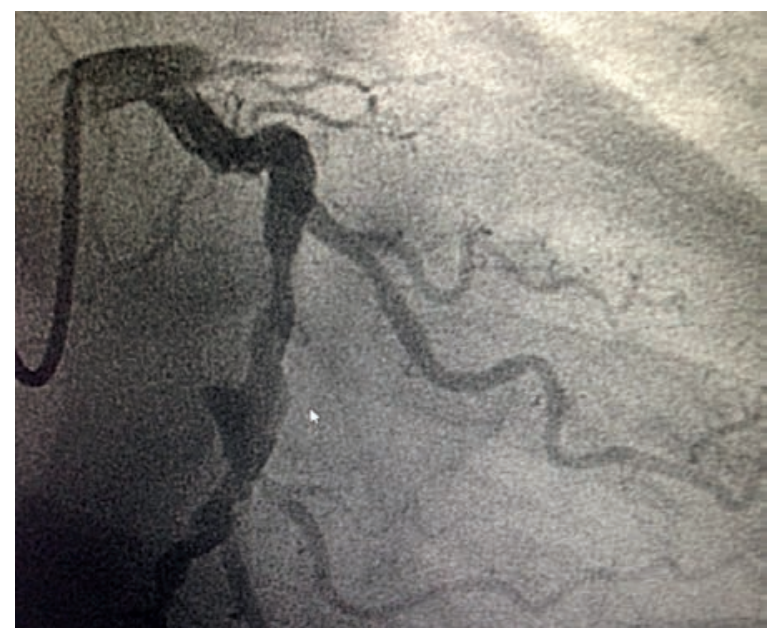

Figura 1. Cinecoronariografía, que informó: tronco de la coronaria izquierda de mediano calibre, permeable, sin lesiones; arteria descendente anterior (ADA) ocluida en su tercio proximal; arteria circunfleja ( $A C X)$ de gran calibre, tipo ectásica, dominante, permeable, sin lesiones; arteria coronaria derecha (ACD) hipoplásica, permeable, sin lesiones.

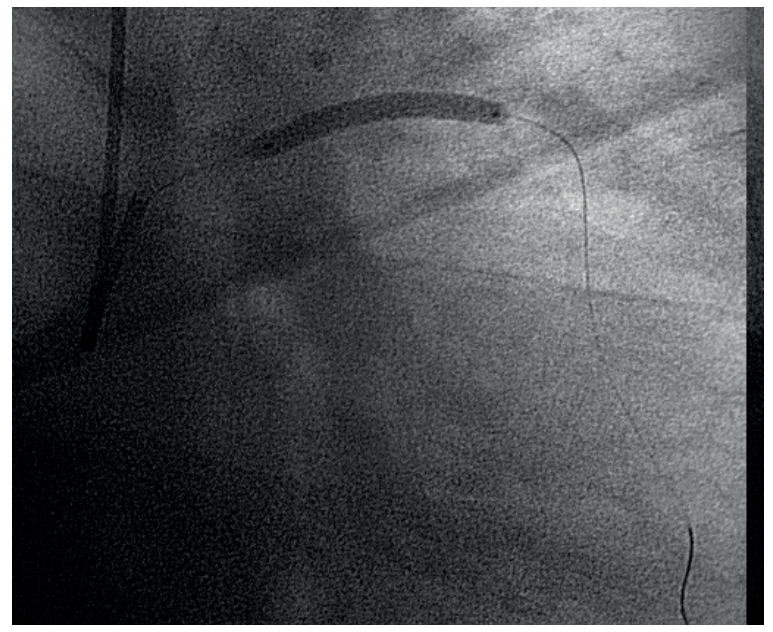

Figura 3. Se impactó stent $3.5 \times 38$ (Waltz $\left.{ }^{\circledR}\right)$ en el sitio de la lesión, impactándolo a 20 atm.

\section{DISCUSIÓN}

Los aneurismas coronarios se definen como una dilatación localizada del vaso que excede en 1,5 veces el diámetro de los segmentos vecinos. La aterosclerosis es la enfermedad más asociada, aunque se han descrito los de origen congénito, los asociados a la enfermedad de Kawasaki, tejido conectivo, enfermedades infecciosas o secundarios al intervencionismo endovascular. Es más frecuente en hombres en una proporción de 4:1 y su localización es, en orden de mayor a menor frecuencia, $\mathrm{ACD}, \mathrm{ACx}, \mathrm{ADA}^{2}$. En nuestro paciente se observó lesión en descendente anterior, lo cual constituye un caso poco frecuente.

$\mathrm{La}$ frecuencia con que se diagnostica ha aumentado de forma significativa, principalmente por medio de la angiografía coronaria y con el uso de métodos diagnósticos no invasivos como ecografía, tomografía, resonancia y gammagrafía ${ }^{3}$. La afectación ateroesclerótica podría ser la causa del desarrollo de aneurismas coronarios por varios mecanismos: inicialmente la formación de la placa de ateroma, con lesión de la íntima y la elástica interna con el depósito de lípidos, colágeno, células (macrófagos, linfocitos T, etc.) y proliferación de

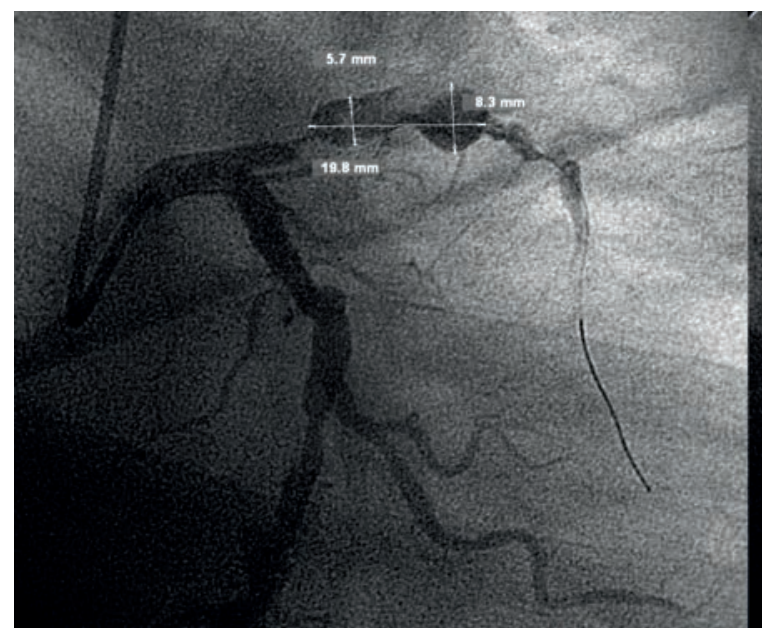

Figura 2. Se decidió realizar angioplastia de urgencia. Se utilizó catéter guía $3.5\left(B X^{\circledast}\right)$, se cateterizó el ostium de la coronaria izquierda, se atravesó una guía floppy 0.014 (Choice ${ }^{\circledR}$ ) posicionándola distalmente en la ADA, se predilató con un balón 2,5x 20 mm (Maverick ${ }^{\circledR}$ ), consiguiendo la apertura del vaso. Se observó aneurisma gigante en la unión del tercio proximal con el tercio medio.

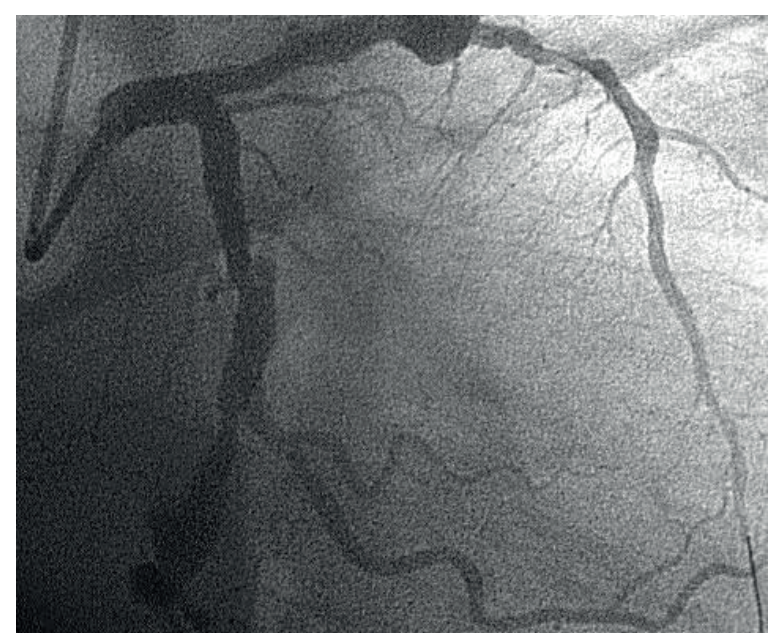

Figura 4. Angiografía de control. Se observó resolución de la lesión, con revas cularización del vaso tratado, sin signos de complicación y flujo final TIMI III.

células musculares lisas. Esta lesión inicial terminaría afectando a las capas media y adventicia con el consiguiente remodeling y la neoformación de vasa vasorum en la placa de ateroma ${ }^{4}$.

Creemos que esta podría ser la etiología en el caso presentado, explicando además la ectasia difusa presente en otras arterias coronarias epicárdicas principales. Se han reportado varias complicaciones importantes durante el curso de la enfermedad, las cuales incluyen: trombosis y embolización distal, isquemia o infarto del miocardio, disección, vasoespasmo, calcificación, fistulización y muy raramente ruptura.

Las opciones terapéuticas consisten en tratamiento quirúrgico, percutáneo o médico.

El tratamiento debe ser individualizado, dependiendo del tamaño, la ubicación, presencia de complicaciones del aneurisma, y también de la clínica del paciente. El limitado número de casos recogidos en la literatura dificulta aún más la toma de decisiones terapéuticas 5 .

En pacientes sintomáticos con estenosis coronarias significativas asociadas al aneurisma, la intervención quirúrgica podría ser la mejor opción, con ligadura del aneurisma y bypass, aunque existen casos en la literatu- 
ra que demuestran la factibilidad, seguridad y eficacia de los stents recubiertos con PTFE para su tratamiento. En pacientes asintomáticos, algunos grupos recomiendan tratamiento conservador mediante un exhaustivo control de los factores de riesgo, antiagregación, asociando anticoagulación oral.

Para la complicación del aneurisma coronario con infarto por trombosis, como en el caso anteriormente expuesto, se sugiere terapia de revascularización endovascular con stents

\section{BIBLIOGRAFÍA}

1. Carvajal CA, Mor JD. Coronary artery aneurysm. Revista Colombiana de Cardiología 85. Julio/Agosto 2005. http://www.oalib.com/paper/1037500\#.U40-fnalf5M.

2. Mata KM, Fernandes CR, Floriano EM, et al. Coronary Artery Aneurysms: An Update, Novel Strategies in Ischemic Heart Disease, Dr. Umashankar Lakshmanadoss (Ed.) (2012). http://cdn.intechopen.com/pdfs-wm/29906.pdf.

3. Cohen P, O'Gara PT. Coronary Artery Aneurysms. A Review of the Natural History, Pathophysiology, and Management. Cardiology in Review. Volume 16, Number 6 convencionales o con stent recubiertos con PTFE (stent graft). Estos sellan efectivamente el aneurisma, pero tienen mayor incidencia de trombosis subaguda entre el primero y el segundo mes, lo cual podría deberse a una endotelización más tardía con el $\mathrm{PTFE}^{6}$. En el caso anteriormente presentado, se optó por stent convencional, consiguiendo la revascularización del vaso, sumando al tratamiento médico antiisquémico/antiagregante habitual la anticoagulación por vía oral, con excelente evolución.

4. Gutowski T, Tannenbaum AK, Moreyra AE. Vasospasm in a coronary artery aneurysm. Cathet. Cardiovas. Diagn. 1991;22:127-129. http://onlinelibrary.wiley.com/doi/10.1002/ccd.1810220212/abstract.

5. Syel M, Lesch M: Coronary artery aneurysm: a review. Prog Cardiovasc Dis 1997:40:77-84.

6. Stankovic G, Colombo A, Presbítero P, van den Branden F, Inglese L, Cernigliaro C, et al:Randomized evaluation of polytetrafluoroethylene-covered stent in saphenous vein grafts: the Randomized Evaluation of polytetrafluoroethylene COVERed stent in Saphenous vein grafts (RECOVERS) Trial. Circulation 2003;108(1):37-42. 